\title{
Gene set enrichment analysis for genome- wide DNA methylation data
}

\author{
Jovana Maksimovic ${ }^{1,2,3}$, Alicia Oshlack ${ }^{1,4,5}$ and Belinda Phipson ${ }^{1,2^{*}}$ (D)
}

\author{
* Correspondence: Belinda. \\ Phipson@petermac.org \\ ${ }^{1}$ Peter MacCallum Cancer Centre, \\ Melbourne, Victoria 3000, Australia \\ ${ }^{2}$ Department of Pediatrics, \\ University of Melbourne, Parkville, \\ Victoria 3010, Australia \\ Full list of author information is \\ available at the end of the article
}

\begin{abstract}
DNA methylation is one of the most commonly studied epigenetic marks, due to its role in disease and development. Illumina methylation arrays have been extensively used to measure methylation across the human genome. Methylation array analysis has primarily focused on preprocessing, normalization, and identification of differentially methylated CpGs and regions. GOmeth and GOregion are new methods for performing unbiased gene set testing following differential methylation analysis. Benchmarking analyses demonstrate GOmeth outperforms other approaches, and GOregion is the first method for gene set testing of differentially methylated regions. Both methods are publicly available in the missMethyl Bioconductor $\mathrm{R}$ package.
\end{abstract}

Keywords: DNA methylation, Gene set analysis, Differential methylation, Statistical analysis

\section{Background}

DNA methylation is essential to human development, with roughly $3-6 \%$ of all cytosines methylated in normal human DNA [1]. Epigenetic marks can be modified by environmental exposures, and methylation changes are known to accumulate with age. Aberrant methylation patterning is associated with many diseases, which has led to several large studies profiling DNA methylation, such as The Cancer Genome Atlas, Encyclopedia of DNA Elements, and numerous epigenome-wide association studies.

Both array- and sequencing-based technologies are available for profiling DNA methylation at a genome-wide scale. However, even though the cost of sequencing has dramatically decreased, the ease and cost-effectiveness of the Illumina human methylation arrays have ensured that the array platforms remain a popular choice. To date, a major focus when analyzing DNA methylation data has been the identification of significantly differentially methylated CpG sites between groups of samples in a designed experiment. There are many well-established analysis methods that perform normalization and statistical testing for this purpose, including publicly available software packages, such as limma [2], minfi [3], missMethyl [4], methylumi [5], wateRmelon [6], ChAMP [7], RnBeads [8, 9], Harman [10], and ENmix [11]. It is well established that methylation of CpG sites is spatially correlated along the genome [12] and that long tracks of differential methylation are often more biologically meaningful

(c) The Author(s). 2021 Open Access This article is licensed under a Creative Commons Attribution 4.0 International License, which permits use, sharing, adaptation, distribution and reproduction in any medium or format, as long as you give appropriate credit to the original author(s) and the source, provide a link to the Creative Commons licence, and indicate if changes were made. The images or other third party material in this article are included in the article's Creative Commons licence, unless indicated otherwise in a credit line to the material. If material is not included in the article's Creative Commons licence and your intended use is not permitted by statutory regulation or exceeds the permitted use, you will need to obtain permission directly from the copyright holder. To view a copy of this licence, visit http://creativecommons.org/licenses/by/4.0/. The Creative Commons Public Domain Dedication waiver (http://creativecommons.org/publicdomain/zero/1.0/) applies to the data made available in this article, unless otherwise stated in a credit line to the data. 
than differences at individual CPG sites [13]. This has led to region-based analyses, with Bioconductor R packages such as Probe lasso [14], bumphunter [15], DMRcate [16], $m C S E A$ [17], and DMRforPairs [18] developed specifically for this purpose.

Once differential methylation analysis between groups of samples has been performed, there may be a long list of significant $\mathrm{CPG}$ sites or regions for the researcher to interpret. A popular approach to gain a more systems-level understanding of the changes in methylation is to examine which gene pathways may be enriched for differential methylation in the experiment. This approach was popularized in the analysis of gene expression microarrays and RNA-sequencing (RNA-Seq) data, with one of the first methods, GSEA, published in 2005 [19]. Since then, a number of gene set testing methods have been developed (e.g., Yaari et al. [20], Wu et al. [21], and Wu and Smyth et al. [22]), all of which are specific to gene expression data, with the GOSeq method [23] developed specifically to account for gene length bias in RNA-seq data.

Methylation, however, is a DNA mark that can occur anywhere on the genome and is not as directly related to genes as expression data. Therefore, a methylation-specific issue in performing gene set testing is how to assign differentially methylated features to genes. Thus far, there are very few gene set testing methods designed specifically for DNA methylation data, and often ad hoc approaches are taken. Only two other methods, ebGSEA [24], and methylGSA [25], have been proposed for gene set enrichment analysis (GSEA) of methylation array data. MethylGSA is an R Bioconductor package that contains several different gene set testing approaches: mRRA, which adjusts multiple $\mathrm{p}$ values for each gene by Robust Rank Aggregation followed by either over-representation analysis (ORA) or functional class scoring in combination with GSEA, and mGLM, which is an extension of GOglm, implementing a logistic regression to adjust for the number of probes in the enrichment analysis [26]. The ebGSEA method uses a global test to rank genes, instead of CpGs, based on their total level of differential methylation; enrichment of gene sets is then calculated from the ranked gene list using either a Wilcoxon Test (WT) or Known Population Median Test (KPMT) [24]. Both ebGSEA and the methylGSA methods use individual CpG probebased differential methylation features, and we are presently not aware of any methods for performing gene set testing for differentially methylated regions.

Here we present GOmeth and GOregion to perform gene set analysis in the context of DNA methylation array data for differential methylation of CpG sites and regions, respectively. The key aspect of our methods is the ability to take into account biases inherent in the data, which relate to how differentially methylated probes are annotated to differentially methylated genes, that can then be assigned to a gene set. Specifically, measured CPG sites are not distributed evenly across the genome, and we and others show that genes that have more CpG sites measured across them are more likely to be detected as differentially methylated compared to genes that have fewer measured $\mathrm{CpG}$ sites. Our original implementation of GOmeth addressed this bias [4]. In addition, approximately $10 \%$ of gene-annotated $\mathrm{CpGs}$ are assigned to more than one gene, violating assumptions of independently measured genes. Our new and improved implementation of GOmeth considers both of these biases in our approach for detecting enriched pathways. Further to this probe-wise analysis, we developed the GOregion method, which takes these biases into account when performing gene set testing following a regionbased analysis. Finally, we have implemented new functionality allowing users to 
restrict gene set testing to only the probes annotated to specific genomic features, such as probes in promoters of genes.

In this paper, we have evaluated the performance of our methods on real and simulated data, as well as comparing to other available methods across a variety of datasets. We found that our methods were the best statistical and computational performers across a variety of comparisons and gene set testing collections. Our methods are publicly available in the Bioconductor R package, missMethyl. All of the analysis performed in this paper can be found at the following website: http://oshlacklab.com/methylgeneset-testing/. The GitHub repository associated with the analysis website is at: https://github.com/Oshlack/methyl-geneset-testing.

\section{Results}

\section{Composition biases of $450 \mathrm{~K}$ and EPIC arrays}

Consider the scenario where we have performed differential methylation analysis on individual CpG sites. In order to perform gene set enrichment analysis based on the results from a probe-wise differential methylation analysis, we need to annotate each probe on the array to a gene. One approach for gene set testing is to simply call a gene differentially methylated if at least one CpG site associated with that gene is significantly differentially methylated, and this has been used in many previous analyses (e.g., Zhang et al. [27], Phipson and Oshlack [28]). The problem with this approach is that the numbers of $\mathrm{CpG}$ sites measured across each gene varies significantly across the genome (Fig. 1A, Additional file 1: Fig. S1A). For the 450K array, the minimum number of CpGs measured per gene is 1 and the maximum is 1299 with a median of 15 , based on the IlluminaHumanMethylation450kanno.ilmn12.hg19 annotation package. For the EPIC array, the numbers of CpGs measured across genes ranges from 1 to 1485 (median $=20$, IlluminaHumanMethylationEPICanno.ilm10b4.hg19 annotation package). Genes that have larger numbers of CpGs measured are more likely to be called differentially methylated when comparing B cells vs natural killer cells (Fig. 1B), and this holds true for the majority of data sets. This bias towards genes with more measured CpG sites can in turn influence the probability of a gene set being called significantly enriched, as some gene sets contain genes with more than the average number of CpGs, and some have genes with fewer measured CpGs (Fig. 1C). In this paper, we refer to this particular source of bias as "probe-number bias".

Approximately $70 \%$ of the probes on the EPIC array are annotated to at least one gene ( $74 \%$ for $450 \mathrm{~K}$ array). However, another annotation issue, which is more subtle, is that a single $\mathrm{CpG}$ may be annotated to more than one gene as the gene regions overlap on the genome. While the majority of CpGs with gene annotations are associated with only one gene $(329,365 / 359,832=92 \%$ for $450 \mathrm{~K}, 554,221 / 607,820=91 \%$ for EPIC arrays), there are still a large number of CpGs annotated to 2 or more genes (Fig. 1D, Additional file 1: Fig. S1B). This can cause issues as the measurements of differentially methylated genes are not independent. If we use every gene associated with a single $\mathrm{CpG}$, we risk counting a single significant $\mathrm{CpG}$ site multiple times when including the genes as enriched in a gene set of interest. If these genes were evenly distributed across the GO categories, this may not be an issue. However, genes that are close in genomic proximity can be functionally related and be present in a single GO category. An 


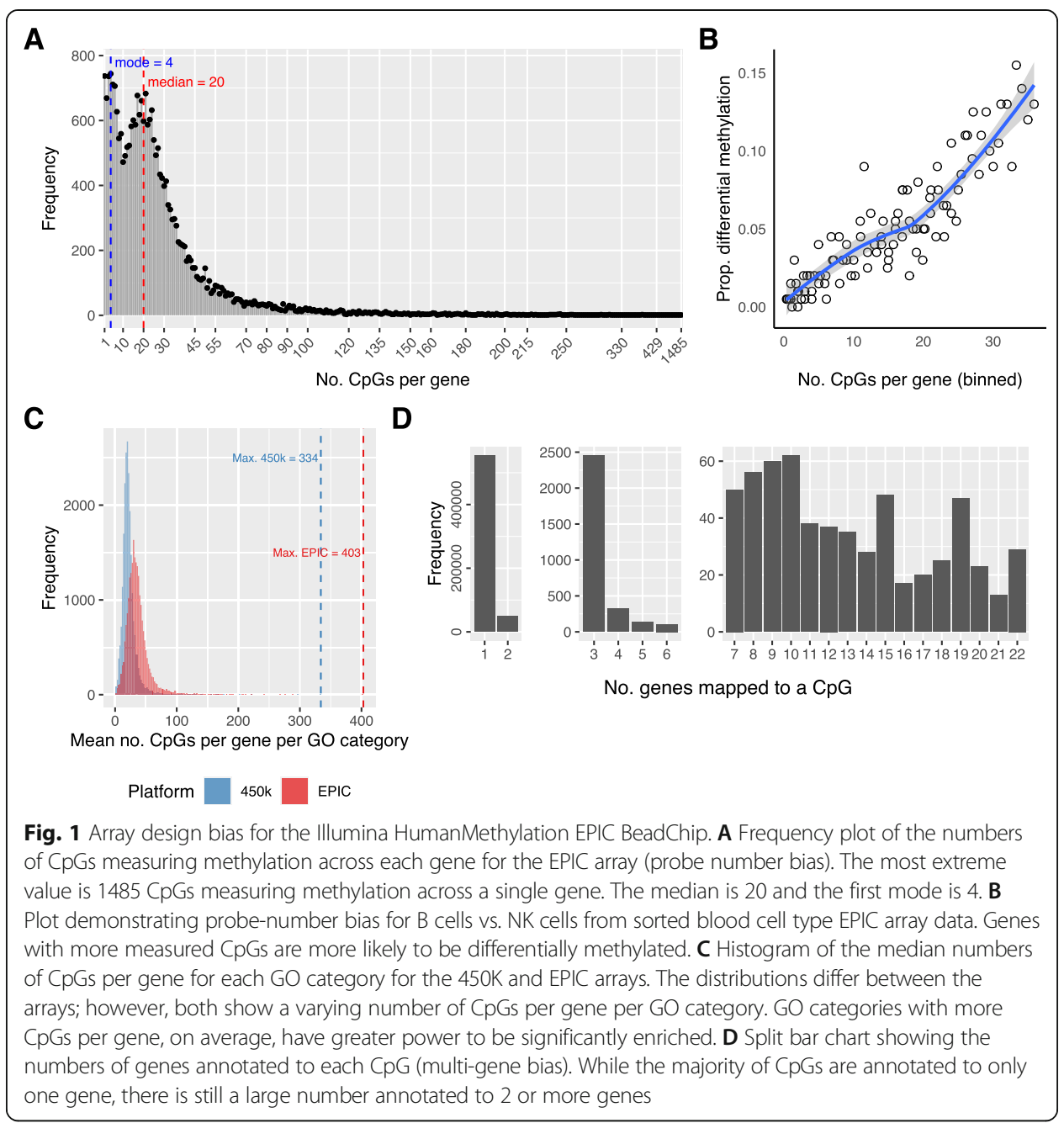

extreme example of this is cg17108383 which is annotated to 22 genes, all belonging to the protocadherin gamma gene cluster (Additional file 1: Fig. S2). All 22 of these genes are present in the GO category "GO:0007156: homophilic cell adhesion via plasma membrane adhesion molecules", which contains a total of 129 genes. If each of these significant genes are included when performing a hypergeometric test for enrichment of the GO category, then for this single significant $\mathrm{CpG}$ site, the overlap between the differentially methylated genes and the genes in the gene set is increased by 22 , and this GO category will appear significantly enriched. Unfortunately, this is not an isolated occurrence. For the EPIC array, gene ontology (GO) enrichment analysis on the 53,599 CpGs that are annotated to at least 2 genes results in 114 significantly enriched GO categories (Holm's adjusted $p$ value cut-off < 0.05) (Additional file 2: Table S1). Restricting to CpGs that are annotated to at least 3 genes results in significant enrichment of 56 GO categories (Holm's adjusted $\mathrm{p}$ value cut-off $<0.05$ ), which are mostly related to processes involved in transcriptional regulation (Additional file 2: Table S2).

We refer to this newly identified source of bias as "multi-gene bias". In order to reduce false positives, it is important to take this multi-gene bias into account when calculating the intersection between differentially methylated genes and the genes in each 
gene set. One approach for dealing with multi-gene bias is to simply randomly select one gene to be represented by the CpG, but this approach risks losing valuable information by ignoring the remaining associated genes. We include this multi-gene bias in our statistical framework for gene set testing using a weighting strategy (Fig. 2) and ensure significant CpGs are only counted once, at most.

\section{GOmeth performs gene set testing on differentially methylated CpG sites}

Our method for gene set testing performs enrichment analysis of gene sets while correcting for both probe-number and multi-gene bias in methylation array data. The detailed statistical approach is outlined in the "Methods" section. This method was inspired by GOSeq [23]. The GOSeq method was designed to account for the fact that longer genes have more sequencing read counts compared to shorter genes, and hence have more power to be statistically significantly differentially expressed. Similarly, we see that genes with a larger number of measured CpGs have a higher probability of differential methylation (Fig. 1B). To account for this bias, GOmeth empirically estimates the probability of differential methylation of a gene as a function of the number of CpGs. In generating the trend, we adjust the number of $\mathrm{CpGs}$ in a gene to account for those that are assigned to multiple genes by using a fractional count. As the probability of differential methylation is calculated empirically from the data, this trend may look different between datasets, but generally, we always observe a strong positive trend. From the empirical trend, the odds of differential methylation for each gene set or gene ontology category is calculated based on the average probabilities of genes in the set. The intersection of the number of differentially methylated genes in the gene set of size $g$ also needs to be defined. Rather than just assigning all genes with a differentially methylated CpG equally, we also take into account the CpGs annotated to multiple genes in this step. Specifically, we assign a weight, $w_{i j}$, to each $\mathrm{CpG}$ that is the reciprocal of the number of genes annotated to the CpG (Fig. 2). We then sum all the CpG weights assigned to a gene and add one to the overlap if the weights are greater than one and $\operatorname{sum}\left(w_{i j}\right)$ otherwise. If all CpGs were annotated to exactly one gene, then the weight, $w_{i j}$, would be one for every $\mathrm{CpG}$, and the overlap would be the number of genes

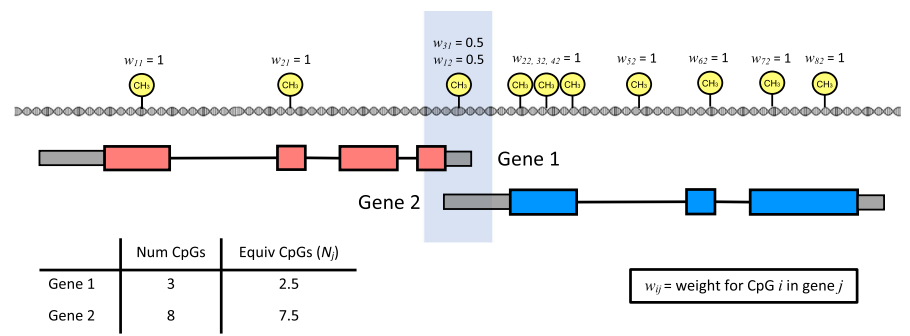

Fig. 2 Overview of how probe number and multi-gene bias is taken into account in GOmeth. CpGs are not evenly spaced throughout the genome. Gene 1 has methylation measured at three CpGs, and gene 2 has methylation measured at eight CpGs. The CpG shaded in blue is an example of a shared genomic location between genes 1 and 2, with one CpG measuring the methylation status for two genes, and is thus not independently measured. By calculating a weight for each CpG inversely proportional to how many genes that $\mathrm{CpG}$ is annotated to, we can calculate the equivalent numbers of CpGs measured across each gene and ensure the enrichment statistic for Wallenius' noncentral hypergeometric test is not artificially inflated due to multi-gene annotated CpGs 
with a significant $\mathrm{CpG}$. However, if a significantly differentially methylated $\mathrm{CpG}$ is annotated to 2 genes, the CpG is assigned a weight of 0.5 to each gene. If no other significant CpGs are annotated to that particular gene, then the gene will contribute a "count" of 0.5 to the intersection statistic for the Wallenius' noncentral hypergeometric test. Thus, if both genes are present in the same gene ontology category, they will contribute a total count of at most one to the intersection statistic. For genes with multiple significant CpGs that may include several multi-gene associated CpGs, the maximum total count a gene can contribute to the intersection statistic is 1 .

Next, a test based on Wallenius' noncentral hypergeometric distribution is performed for each gene set or gene ontology category, incorporating the odds that a gene set is more or less likely to be enriched based on the probe-number bias of the experiment with the intersection statistic defined above [4].

We have implemented our approach to gene set testing with two different functions in the missMethyl Bioconductor R package, "gometh," and "gsameth." The difference between the two functions is minimal, with gometh specifically testing for enrichment of gene ontology (GO) categories from the GO.db annotation package, or KEGG pathways from the KEGG.db annotation package. The gsameth function is a more general version of gometh, where the user can supply any list of gene sets to be tested. In addition, the gometh and gsameth functions allow the set of significantly differentially methylated CpGs to be restricted to genomic regions of interest such as promoters or gene bodies, as these may interrogate different biological pathways.

\section{Improved type I error rate control with GOmeth}

We first tested the performance of GOmeth by randomly sampling sets of CpG probes from the EPIC and 450K array annotation that we designated as differentially methylated and running gene ontology analyses. Under these null scenarios, we would not expect to see significant enrichment of any GO categories. We randomly selected 100 sets each of 50,100, 500,1000,5000, and 10,000 CpGs as "significantly" differentially methylated based on the 450K and EPIC annotation. We tested for enrichment of gene ontology sets for each simulation and calculated the number of GO categories that were significant at a p value threshold of 0.05 . For a test to correctly control the type I error rate, we expect $5 \%$ or fewer GO categories to have significant $\mathrm{p}$ values for random data. We compared the three testing options available in GOmeth: the hypergeometric test with no bias corrections ("HGT"), Wallenius' noncentral hypergeometric test taking into account probe-number bias only ("HGT-mod") and GOmeth, which takes into account both probe-number and multi-gene bias (Fig. 3A \& B, Additional file 1: Fig. S3A \& B). Under these simulation conditions, we were unable to compare to the methylGSA methods and ebGSEA since these tests require $M$ values or $\beta$ values as input rather than just the list of significant probes.

Figure 3A summarizes the type I error rates for our methods across the varying sets of randomly selected CpGs sampled from the EPIC array. As the numbers of differentially methylated CpGs increased, we noted that the hypergeometric test reported too many significant GO categories, particularly for more than $500 \mathrm{CpGs}$. Clearly, taking into account the probe-number bias makes the biggest correction to false discoveries, with HGT-mod and GOmeth maintaining the correct type I error rate. Correcting for 


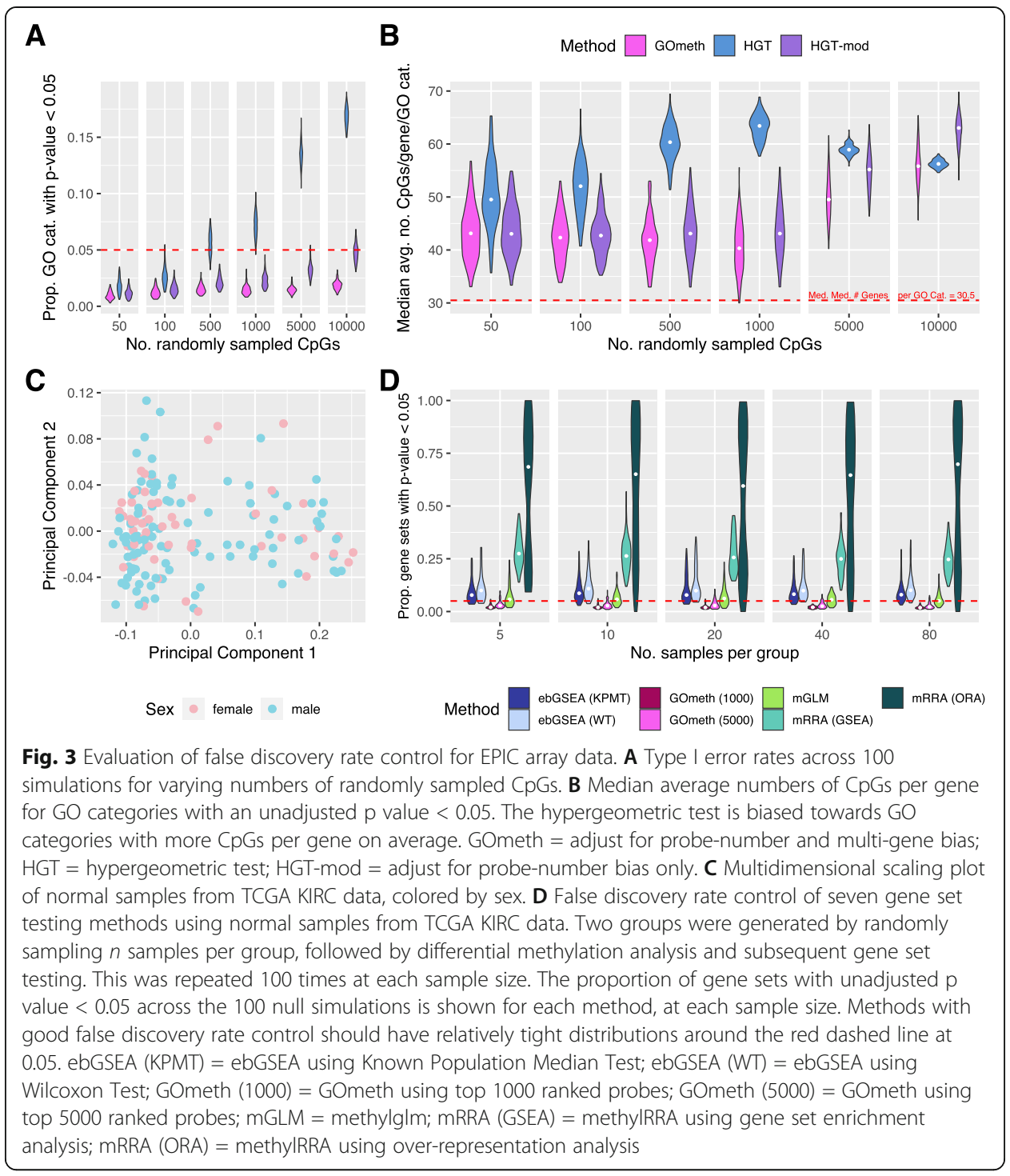

multi-gene bias (GOmeth) further reduced the numbers of significantly enriched GO categories. We did notice that not accounting for the multi-gene bias led to an increase in false discoveries as the numbers of significant CpGs increased. A similar trend was observed for simulations based on the $450 \mathrm{~K}$ array (Additional file 1: Fig. S3A).

Of the significant GO categories reported by each method, we found that the hypergeometric test was more biased towards GO categories with more CpGs measured per gene on average (Fig. 3B, Additional file 1: Fig. S3B). HGT-mod and GOmeth reported significant categories that had a wide range of CpGs per gene on average. Based on the results of these simulations, we selected GOmeth taking into account both probenumber and multi-gene bias as the best option to use for further analysis, even though it is quite a conservative test in this particular simulation scenario.

The prior simulations are limited in that they only generate a random set of CpGs to test for over-representation of gene sets. However, previously published methods, ebGSEA and the methylGSA methods, require differential methylation measurements ( $M$ values or $\beta$ values) as input. In order to compare the type I error rate control of 
GOmeth with ebGSEA and methylGSA, we analyzed the normal samples from the TCGA $450 \mathrm{~K}$ array kidney renal cell carcinoma (KIRC) dataset (Fig. 3C), as well as the normal samples from the TCGA $450 \mathrm{~K}$ breast invasive carcinoma (BRCA) dataset (Additional file 1: Fig. S3C). For both datasets, we took a resampling approach whereby we randomly assigned samples to one of two "groups" and varied the sample size per group $(\mathrm{n}=5,10,20,40,80)$. We then performed differential methylation analysis between the two artificial groups, followed by gene set testing using the available methods: mGLM, mRRA (ORA), mRRA (GSEA), ebGSEA (WT), ebGSEA (KPMT), GOmeth with top $1000 \mathrm{CpGs,}$ and GOmeth with top 5000 CpGs. While the ebGSEA methods are available in the ChAMP $\mathrm{R}$ Bioconductor package, we used a more flexible implementation available in the $e b G S E A \mathrm{R}$ package on GitHub. We defined the input for GOmeth as either the top 1000 or top 5000 most highly ranked differentially methylated CpGs even though the probes did not reach statistical significance. This allowed us to calculate the proportions of gene sets that were significantly enriched for each of the methods, where "significant" is defined as a p value less than 0.05 . We repeated these steps 100 times at each sample size. In this scenario, where there are no true biological pathways differentially methylated, we expect $5 \%$ or fewer gene sets to be significantly enriched. We used the 8567 gene sets from the Broad's Molecular Signatures Database (MSigDB) available in the ChAMP package to evaluate the false discovery rate. In addition, to ensure that the methods in the methylGSA package produced reasonable output, we limited the size of the gene sets to those with at least 5 and at most 5000 genes in the set. If these additional constraints are not included, the methylGSA methods mGLM and mRRA (ORA) produced results that were heavily biased towards reporting very small gene sets as highly significant. Furthermore, mRRA (ORA) was also biased towards ranking large gene sets very highly, if they were not filtered out (Additional file 1: Fig. S3E).

In total, we compared seven different variants of the gene set tests: GOmeth with top $1000 \mathrm{CpGs}$, GOmeth with top $5000 \mathrm{CpGs}$, the three testing frameworks in methylGSA and the two tests in ebGSEA. In general, varying the sample size did not make a difference to the results, with consistent patterns observed for all sample sizes, across the two datasets (Fig. 3D, Additional file 1: Fig. S3D). The worst performing test was mRRA (ORA), which had a median proportion of significantly enriched gene sets of at least 0.6 (or a median of 5388 enriched gene sets, across the 5 sample sizes). mRRA (GSEA) also performed poorly, with a median proportion of significantly enriched gene sets of at least 0.25 (or a median of 2117 enriched gene sets, across the 5 sample sizes). However, mGLM correctly controlled the false discovery rate at 0.05 . The two variants of ebGSEA had slightly greater than 0.05 median proportion of false discoveries although their results were more variable across the 100 simulations at each sample size, with some simulations showing large numbers of false positives. GOmeth, using both top 1000 and 5000 CpGs, showed highly consistent performance, with median proportions of false discoveries $<0.05$ (with a median of 180 and 278.5 enriched gene sets, respectively, across the 5 sample sizes), suggesting that GOmeth correctly controls for false discoveries. The performance of the seven methods was highly consistent across both the KIRC and BRCA datasets. 


\section{Application to blood cell type EPIC data}

Following our simulation studies, we wanted to test the performance of GOmeth, ebGSEA, and the methylGSA methods on real data that contained differential methylation. We used a publicly available dataset of flow sorted blood cell types profiled on Illumina Infinium HumanMethylationEPIC arrays (GSE110554) [29]. Cell types are easily distinguished based on methylation patterns (Fig. 4A). We chose to perform our differential analysis and gene set testing on three independent pair-wise comparisons of cell types with varying numbers of differentially methylated probes: (1) CD4 vs CD8 T cells, (2) monocytes vs neutrophils, and (3) B cells vs natural killer (NK) cells (Fig. 4B). Differential methylation was performed using TREAT [30], and CpGs were defined as significantly differentially methylated if they had false discovery rates $<0.05$ and $\Delta \beta$ cut-off of $\sim 10 \%$ (corresponding to $\Delta \mathrm{M} \sim 0.5$ ). Following the differential methylation analysis, we tested enrichment of GO sets and KEGG pathways using hypergeometric tests, GOmeth, the three methylGSA methods, and the two ebGSEA tests. Again, we limited the gene sets to those with a minimum of 5 and a maximum of 5000 genes for methylGSA. For input to GOmeth, we used either the statistically significant CpGs at FDR $<0.05$ (CD4 vs CD8 T cells) or the top 5000 most highly ranked CpGs for comparisons with more than 5000 significant CpGs (B cells vs NK cells and monocytes vs neutrophils).

To evaluate the significant gene sets in a systematic way we took two approaches. First, we identified all immune categories in the GO database, as these are expected to be highly enriched when comparing different blood cell types. We therefore defined "true positive" GO categories as all the child terms under the parent GO category "immune system response" (GO:002376) from AMIGO 2 (http://amigo.geneontology.org/ amigo/term/GO:0002376). We then counted how many of these immune sets were present in the top ranked gene sets for each method. We similarly defined true positives for the KEGG pathways by identifying all pathways belonging to the following categories: immune system, immune disease, signal transduction, signaling molecules, and interaction (https://www.genome.jp/kegg/pathway.html). The second approach we took to evaluate the different methods was by analyzing a publicly available RNA-Seq dataset comparing the same sorted blood cell types (GSE107011; SRP125125) [31, 32]. We performed differential expression analysis and gene set testing on the expression data and defined the top 100 significantly enriched gene sets from the RNA-Seq analysis as the "truth" (Additional file 1: Fig. S4A-D).

For GO categories, GOmeth consistently performed the best with the highest numbers of top ranked categories overlapping with truth sets across the three comparisons (Fig. 4C-D, Additional file 1: Fig. S5-6). For KEGG pathways, the differences between the methods were not as clear, with mGLM, ebGSEA, and GOmeth generally performing well. The mRRA (GSEA) and mRRA (ORA) methods had highly variable performance (Additional file 1: Fig. S7). For the monocyte vs neutrophil comparison, the ebGSEA methods had more power to detect significant gene sets (Additional file 1: Fig. S7E).

Next, we examined the top 10 ranked terms for each of the seven gene set testing methods (Fig. 4D, Additional file 1: Fig. S5-7). For the B cells vs NK cells, we noted that the hypergeometric test, and to a lesser extent, the two ebGSEA methods, tended to have very large, non-specific GO categories most highly ranked, with "protein binding," 


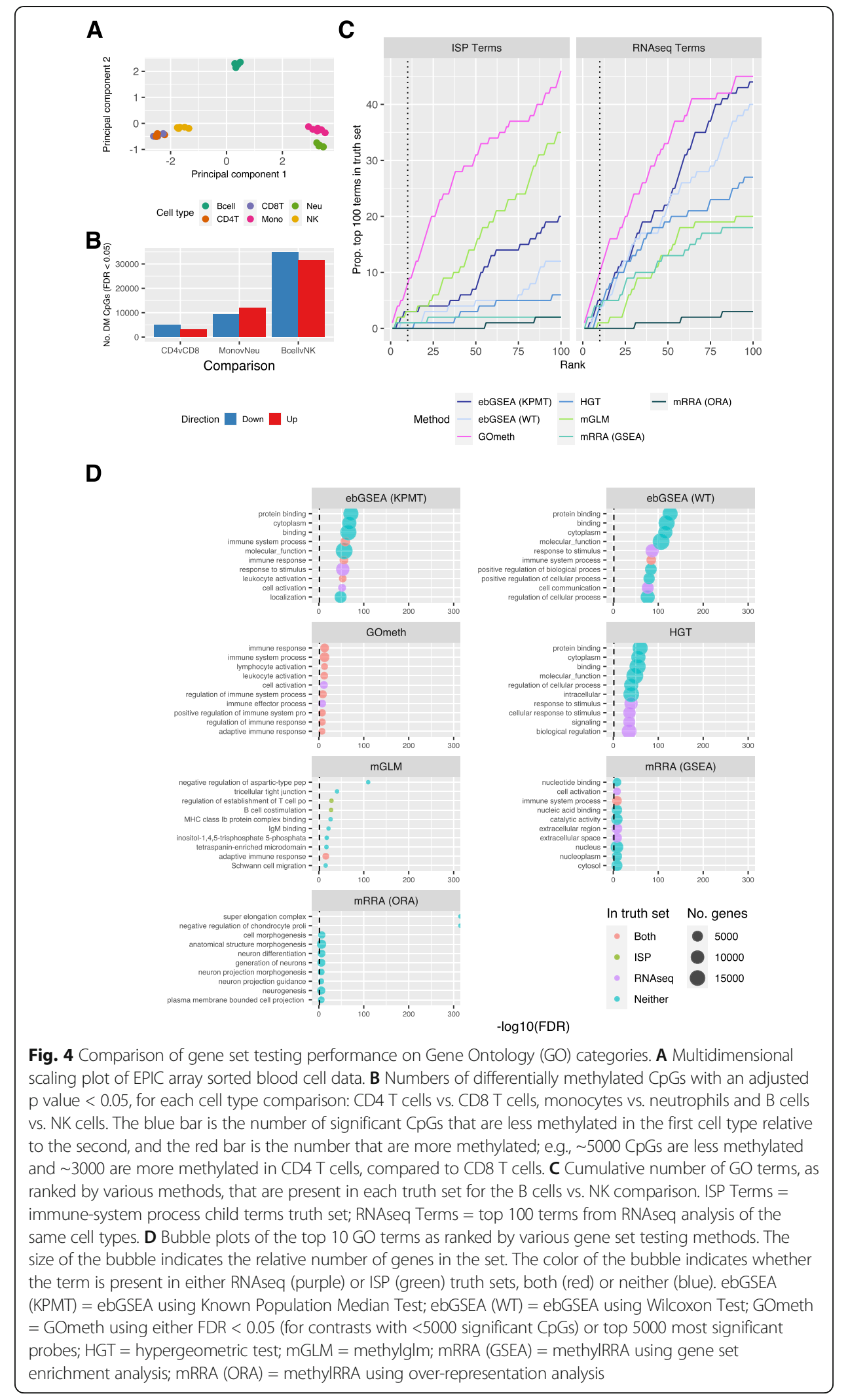


"cytoplasm", and "molecular function" in the top 10 (Fig. 4D). The top 10 enriched GO categories for GOmeth were more biologically relevant, with immune specific gene set tests highly enriched (for example "leukocyte activation" and "lymphocyte activation"). All of the top 10 terms for GOmeth were included in at least one "truth" set. For the methylGSA methods, mGLM appeared to have more immune specific categories in the top 10 (e.g., "adaptive immune response" and "regulation of leukocyte cell-cell adhesion") compared to mRRA (GSEA) and mRRA (ORA). The results for mRRA (ORA) and mRRA (GSEA) were more difficult to interpret with none of the top $10 \mathrm{mRRA}$ (ORA) results included in either truth set. A similar pattern was observed for the CD4 vs CD8 T cells and monocyte vs neutrophil comparisons, with GOmeth consistently ranking more immune specific terms in the top 10 (Additional file 1: Fig. S5B and 6B).

\section{Application to B cell development $450 \mathrm{~K}$ data}

We applied the seven different gene set testing methods to a developing pre-B cell dataset which measured methylation using the $450 \mathrm{~K}$ array and had matched gene expression captured using Affymetrix arrays. Four populations of B cell developmental stages were isolated from human fetal bone marrow, from 8 individuals. We focused on the comparison between Stage 1 (multipotent progenitor cells) and Stage 2 (pre-B cell I) and analyzed the methylation data taking a similar approach as described above (Additional file 1: Fig. S8A, B). For the gene expression dataset, background correction and normalization were performed using the RMA algorithm [33, 34], low intensity probes were filtered out, and differential expression analysis was performed with limma (Additional file 1: Fig. S4E-G). We created the gene expression truth sets by selecting the top 100 gene sets for GO and KEGG pathways identified using the "goanna" and "kegga" functions in limma. We also used the immune related sets from GO and KEGG as previously described, as alternative truth sets.

For GO categories, GOmeth was the top performer, with ebGSEA (KPMT) performing consistently well across both truth sets (Additional file 1: Fig. S8C, D). The methylGSA methods did not perform as well on this dataset, with mGLM the best of the three. For KEGG pathways, all methods performed similarly (Additional file 1: Fig. S9A); however, the gene sets were not very highly statistically significant (Additional file 1: Fig. S9B). For the top 10 ranked GO categories, GOmeth was the only method that had all 10 present in either truth set (Additional file 1: Fig. S8D). For KEGG pathways, $8 / 10$ pathways for GOmeth were present in either truth set and the top ranked pathway for GOmeth was "hematopoietic cell lineage" (Additional file 1: Fig. S9B).

We generally found, across all dataset comparisons and gene set ensembles, that the hypergeometric test, mRRA (ORA), and mRRA (GSEA) tended to rank large, non-specific categories very highly, with GOmeth ranking more biologically relevant gene sets in the top 10. For the two ebGSEA methods, ebGSEA (KPMT) appeared to perform better than ebGSEA (WT); however, for dataset comparisons with a large number of significant probes, the ebGSEA methods tended to rank large, non-specific categories more highly. For comparisons with fewer significant probes, ebGSEA (KPMT) showed a good performance with more power to detect significant enrichment of gene sets. The mGLM method was the best performer of the three methods available from methylGSA. 


\section{Comparing compute time between gene set testing methods}

While the mGLM and ebGSEA methods generally perform well, computationally, they are the slowest methods to run on a single core ( 48 min for mGLM, $\sim 50$ min for ebGSEA) (Table 1). The run time of ebGSEA and mGLM can be significantly reduced by parallelising the computations. Using 10 cores to complete the analysis of 8567 MSigDB gene sets, ebGSEA took approximately $36 \mathrm{~min}$, and mGLM took $16 \mathrm{~min}$. We also show the run-time results for 10 and 20 cores in Table 1. By comparison, GOmeth is 90 times faster than mGLM and ebGSEA on a single core. mRRA (ORA) is the fastest to run but generally does not perform as well as other methods.

\section{Gene set testing following a region-based analysis}

CpGs are not evenly spaced across the genome and often appear in clusters, e.g., CpG islands [35], and several studies have demonstrated that CpGs in close proximity have correlated methylation levels [12]. Thus, rather than testing individual CpGs, identifying correlated methylation patterns between several spatially adjacent CpGs has been shown to yield more functionally relevant results [13].

Several tools have been published for identifying differentially methylated regions (DMRs) from methylation array data: Probe lasso [14], bumphunter [15], DMRcate [16], $m C S E A$ [17], and DMRforPairs [18]. Depending on the input data, region finding tools can identify several hundred or even thousands of DMRs. They all generally output the location of the region including the chromosome, region start, and region end positions, along with some additional metrics and statistical significance. Some tools attempt to annotate the regions with genes but others do not. Thus, when faced with a long list of DMRs, it is unclear how to interpret the biological significance of the results, and there are no gene set testing tools available for DMRs.

To address this, we have developed GOregion, an extension of GOmeth, that enables gene set testing of DMRs. The "goregion" function tests GO terms and KEGG pathways, while "gsaregion" is a generalized function that accepts any list of gene sets as input. We reasoned that because region detection is inherently dependent on $\mathrm{CpG}$ probe density, DMRs are more likely to be identified in genes with more CpG probes. This trend is observed in the blood cell type and $\mathrm{B}$ cell development data (Fig. 5A, Additional file 1: Fig. S10A, 11A, 14A). To take this bias into account, GOregion utilises the GOmeth testing framework. GOregion accepts a ranged object of DMRs that have been identified by the user's choice of region-finding software. These regions are

Table 1 Average run-time across all contrasts. Gene sets used are Broad MSigDB gene sets from the ChAMP package. All methods were run on a Blade Server: 24 CPUs - Intel(R) Xeon(R) Gold 6126 CPU @ 2.60GHz

\begin{tabular}{llll}
\hline Method & \multicolumn{2}{l}{ Time (minutes) } & $\mathbf{1 0}$ cores \\
\cline { 2 - 4 } & $\mathbf{1}$ core & - & cores \\
\hline mRRA (ORA) & 0.13 & - & - \\
GOmeth & 0.54 & - & - \\
mRRA (GSEA) & 3.04 & 16.12 & 9.86 \\
mGLM & 47.95 & 36.07 & 33.79 \\
ebGSEA & 50.12 & &
\end{tabular}




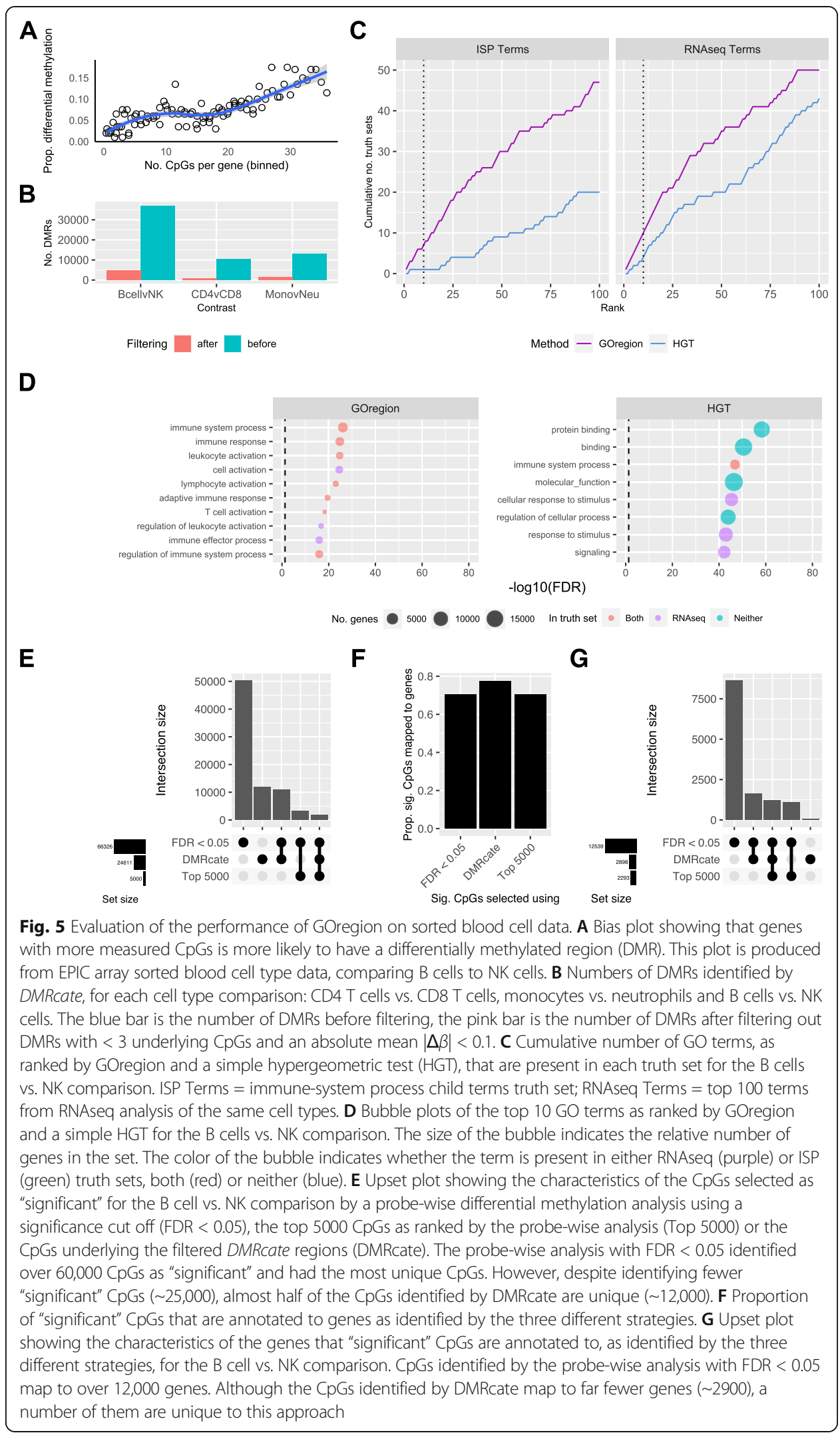


then overlapped with the locations of the CpGs on the Illumina array to identify a set of CpGs underlying the DMRs. These CpG probes are then passed to GOmeth, and GOmeth's existing algorithm is used to test for enrichment of gene sets.

\section{GOregion applied to sorted blood cell type EPIC data}

Using the sorted blood cell EPIC data, we identified DMRs for the same three cell type comparisons using the DMRcate package: (1) CD4 vs CD8 T cells, (2) monocytes vs neutrophils, and (3) B cells vs NK cells. Using default parameters, DMRcate identified 6404, 7176, and 23,210 differentially methylated regions, respectively. We further filtered the DMRs by only including regions containing 3 or more CpGs with an absolute mean $\beta$ value difference greater than 0.1 . This left 789, 1633, and 4723 DMRs, respectively, for downstream analysis (Fig. 5B). We then performed gene set testing of GO categories using GOregion and compared it to a simple approach of overlapping DMRs with known genes and then testing using a HGT.

As previously described, we evaluated the results by counting the numbers of highly ranked immune-related GO terms, and the numbers of highly ranked GO categories identified in the RNA-Seq data analysis of the same cell types. GOregion consistently ranked immune-related and RNA-Seq truth terms more highly than the simple HGTbased strategy (Fig. 5C, Additional file 1: Fig. S10B, 11B). Examining the top 10 most highly ranked GO categories showed that GOregion categories are more specific to immune processes than those identified using the HGT approach (Fig. 5D, Additional file 1: Fig. S10C, 11C). Across all comparisons, GOregion always ranked more truth sets in the top 10 than HGT. For example, the top 10 gene sets ranked by GOregion for the B cells vs NK comparison were highly specific to immune system processes, e.g., "T cell activation," whereas for HGT the sets were very broad and contained thousands of genes, e.g., "protein binding" (Fig. 5D).

We wanted to explore the differences between a region analysis and a probe-wise analysis and how this would affect gene set testing results. GOmeth is highly dependent on the set of significant CpGs provided as input, so we postulated that selecting CpGs using a region-level analysis could be more biologically relevant, in certain circumstances. We compared the numbers of differentially methylated probes and genes that are selected based on a region analysis with DMRCate, a probe-wise analysis using an FDR cut-off $<0.05$, and a probe-wise analysis selecting the top 5000 differentially methylated CpGs. For the B cell vs NK cells comparison, a probe-wise analysis with FDR < 0.05 selects over 60,000 differentially methylated CpGs. More than 50,000 of these CpGs are unique to this approach and not identified with the region approach, whereas DMRCate identifies 24,000 CpGs in DMRs (Fig. 5E). We noted that although DMRCate always captured fewer significant CpGs (Fig. 5E, Additional file 1: Fig. S10D, 11D), a higher proportion of the total significant CpGs was annotated to genes compared with either of the probe-wise approaches (Fig. 5F, Additional file 1: Fig. S10E, $11 E$ ). This results in sets of genes uniquely captured using a region-based analysis (Fig. 5G, Additional file 1: Fig. S10F, 11F).

Comparing GOregion to the two probe-wise approaches using our previously defined truth sets showed that all of the approaches performed similarly well across all the contrasts, except for B cells vs NK cells (Additional file 1: Fig. S12A, B). For that 
comparison, GOregion and the GOmeth probe-wise analysis using the top 5000 CpGs both performed markedly better than GOmeth with significant CpGs selected using FDR $<0.05$ (Additional file 1: Fig. S12A). This is likely due to these significant CpGs being annotated to $>12,000$ genes, resulting in a highly non-specific set of genes as input to GOmeth, whereas DMRCate regions are annotated to just under 3000 genes (Fig. 5G). Examining the terms that were highly ranked by the various approaches, across the different contrasts, revealed that they all tended to be immune specific (Additional file 1: Fig. S12C-E). The exception is the B cells vs NK comparison produced by GOmeth based on CpGs selected at FDR $<0.05$, where the most highly ranked terms were very broad categories such as "cell communication," "signaling", and "plasma membrane part" (Additional file 1: Fig. S12C). Hence, for comparisons which result in very large numbers of significantly differentially methylated CpGs, limiting the set of CpGs used as input for gene set testing, such as performing a region analysis, is important for producing meaningful results.

Region-finding software is itself dependent on numerous parameters and appropriate downstream filtering of results can also be important in identifying the most biologically informative set of DMRs. Using the blood cell type dataset, we demonstrate that different DMR filters affect the downstream GOregion gene set testing results in different ways, depending on the comparison (Additional file 1: Fig. S13). For our dataset, the most important filter to include is a mean $\Delta \beta$ difference cut-off, i.e., the size of the methylation difference. For this particular dataset, we find that a $\Delta \beta$ difference cut-off of 0.1 performs best across all comparisons. For the B cells vs NK cells comparison, we noticed that not filtering with a $\Delta \beta$ cut-off produced very poor results. In contrast, for the monocyte vs neutrophils comparison, a $\Delta \beta$ cut-off of 0.2 was too stringent and resulted in fewer gene sets overlapping with the truth sets (Additional file 1: Fig. S13A, B).

\section{GOregion applied to $B$ cell development $450 \mathrm{~K}$ data}

Using DMRcate, we identified 2151 DMRs when comparing Stage 1 vs Stage 2 B cells and observed a strong probe-number bias trend (Additional file 1: Fig. S14A). Once again, we compared the top ranked GO terms for GOregion and HGT with our two truth sets: (1) the top 100 ranked gene sets from comparing Stage 1 vs Stage 2 B cells in the Affymetrix gene expression data and (2) the immune related terms. GOregion outperformed HGT, with a greater overlap of the top ranked gene sets with both truth sets (Additional file 1: Fig. S14B). Examining the top $10 \mathrm{GO}$ terms showed that GOregion had 7/10 terms present in either truth set, with only 3/10 gene sets overlapping either truth set for HGT. Two of the top $10 \mathrm{GO}$ terms identified by GOregion, but not identified in either truth sets, were related to cytokine production, which is known to be important in B cell development [36]. HGT ranked very broad, nonspecific GO terms very highly (e.g., "molecular function," "protein binding"), whereas GOregion ranked more immune related gene sets in the top 10 (e.g., "leukocyte activation").

We compared the numbers of differentially methylated probes and genes selected using either a region-based analysis, a probe-wise analysis with $\mathrm{FDR}<0.05$, or a probewise approach selecting the top 5000 differentially methylated CpGs (Additional file 1: Fig. S14D-F). For Stage 1 vs Stage 2 developing B cells, 11,597 probes were selected 
using DMRCate compared to only 3148 with a probe-wise analysis with FDR $<0.05$, suggesting that thousands of probes that are not individually significant were underlying regions (Additional file 1: Fig. S14D). A higher proportion of probes captured using DMRcate was annotated to genes compared to the probe-wise analyses (Additional file 1: Fig. S14E), and although DMRcate selected 11,597 probes, these were annotated to only 1664 genes (Additional file 1: Fig. S14F). As we observed in the sorted blood cell type data, the DMRcate-selected CpGs overlapped genes that were unique to the region-based analysis (Additional file 1: Fig. S14F).

For this particular dataset, GOmeth and GOregion performed very similarly with all methods (GOmeth (top 5000), GOmeth (FDR $<0.05)$ and GOregion) showing a similar degree of overlap with the two truth sets (Additional file 1: Fig. S15A). While there were some differences in the top 10 ranked GO terms, they were all involved in immune system processes (Additional file 1: Fig. S15B).

Although we did not filter the DMRs prior to gene set testing with GOregion for this particular dataset, we investigated the effect of filtering on the overlap with the truth sets (Additional file 1: Fig. S15C). Modifying $\Delta \beta$ or the number of CpGs underlying the regions did not have a drastic effect on the overlap with the truth sets, although for the gene expression array terms, the additional filter of $|\Delta \beta|=0.2$ and number of $\mathrm{CpGs}=4$ reduced the number of truth gene sets identified by GOregion.

\section{Discussion}

Gene set testing is a useful tool to gain additional biological insight into the underlying mechanisms in an experiment. Here we present GOmeth for performing gene set testing after a probe-level analysis, and GOregion, a gene set testing method following a region-based analysis. To the best of our knowledge, GOregion is the only method that specifically tests enrichment of gene sets for differentially methylated regions.

Through our investigation of the composition of the 450K and EPIC arrays, we have observed that annotating statistically significant differentially methylated CpGs to genes is not a trivial exercise. For our gene set testing methods, we choose to rely on the annotation provided by Illumina; however, other studies have used alternative approaches, such as defining promoter regions as regions of $+/-2 \mathrm{~kb}$ of the transcription start sites of genes [37].

We have shown that two sources of bias affect gene set testing: probe-number and multi-gene bias and our methods account for both of these. Through the use of simulations and resampling normal samples, we have shown that GOmeth correctly controls the false discovery rate with minimal bias. We applied GOmeth to sorted blood cell types and developing B cell datasets and showed that the top ranked categories are consistently biologically relevant across multiple comparisons. We defined two different types of truth sets based on the information available in the GO and KEGG databases, as well as independently analyzed gene expression datasets. We acknowledge that our truth sets will have shortcomings in that they are unlikely to encompass all the truly enriched pathways, and, particularly in the case of the gene expression data analysis, the choice of gene set testing method is likely to play a role in how gene sets are ranked. Nevertheless, we showed that GOmeth generally outperforms other available methods. Further, we have shown that the probe-number bias affects the probability that a region is called differentially methylated. We therefore developed GOregion to 
perform unbiased gene set testing following a region-based analysis. For the blood cell types and developing B cell dataset, GOregion outperformed a simple hypergeometric testing approach that has been previously used in analyses.

An important consideration when using GOmeth is how many differentially methylated probes to use as input to the method. For comparisons that have tens of thousands of significantly differentially methylated CpGs, in the first instance, we would recommend performing a region-based analysis and using GOregion to perform gene set testing. Another option would be to use GOmeth, but restrict the input CpGs to the top ranked CpGs, with a rule of thumb that the number of input CpGs is less than 10,000. Simply taking a false discovery rate cut-off for the B cells vs NK cell comparison, for example, resulted in too many genes being identified as differentially methylated $(>12$, 000 ), and we found that the gene set testing results were not very specific or biologically meaningful. In our comparisons, we have used the top 5000 differentially methylated probes and this produced good results for the blood cell type dataset. There is additional functionality in GOmeth to restrict the list of significant $\mathrm{CpGs}$ by genomic features, such as "TSS1500," "TSS200", and "Body," for example. This has the effect of decreasing the overall numbers of CpGs to use as input, but potentially retains more biologically meaningful loci.

For users who are not comfortable with setting thresholds to select appropriate CpGs as input to GOmeth following a probe-level analysis, mGLM and ebGSEA are alternative statistical methods that do not require setting cut-offs to select significant CpGs. Generally, when the numbers of significant probes was large, ebGSEA-ranked large, broad GO terms more highly compared to mGLM. However, when the numbers of statistically significant CpGs were fewer than 5000, ebGSEA performed well in our evaluations.

Even though a region-based approach can potentially select fewer CpGs, in our analysis of the sorted blood cell types and developing B cell data, it always identified additional unique CpGs not detected using the probe-wise methods. This is likely because these CpGs were not statistically significant on their own but are identified as part of a region. Thus, given the potential for capturing biologically important CpGs, which may be missed by probe-wise approaches due to their reliance on rankings and significance cut-offs, we suggest that a good quality, region-based analysis can potentially distill more focused gene sets than a probe-level gene set analysis of the same data.

An important feature of GOmeth and GOregion is their flexibility. Unlike the methylGSA methods, GOmeth and GOregion do not require any filtering of gene sets to produce robust results. GOregion is compatible with the results of any software for finding differentially methylated regions which can be expressed as a ranged data object. Both GOmeth and GOregion are computationally efficient and can test a variety of gene sets such as GO categories, KEGG pathways or any list of custom gene sets.

\section{Conclusions}

GOmeth and GOregion are novel statistical methods that account for biases in gene set testing for methylation arrays. We have shown that our methods produce the most biologically meaningful results while controlling the false discovery rate. All of our gene set testing functions is available in the missMethyl Bioconductor R package. 


\section{Methods}

All analysis code presented in this manuscript can be found at http://oshlacklab.com/ methyl-geneset-testing/. The analysis website was created using the workflowr (1.6.2) R package [38]. The GitHub repository associated with the analysis website is at: https:// github.com/Oshlack/methyl-geneset-testing.

\section{Statistical model for GOmeth}

The statistical test for GOmeth and GOregion is based on Wallenius' noncentral hypergeometric distribution, which is a generalized version of the hypergeometric distribution where items are sampled with bias. For GOmeth, we take the following stepwise procedure:

1. For each CpG $i$ annotated to gene $j$, calculate a weight

$$
w_{i j}=\frac{1}{\# \text { genes annotated to } \mathrm{C} p G_{i j}} .
$$

If each CpG is annotated to exactly one gene, then $w_{i j}=1$.

2. Let $i=1, \ldots, I_{j}$ denote the CpGs annotated to gene $j$. Calculate the equivalent number of CpGs measured across gene $j$ as:

$$
N_{j}=\sum_{i=1}^{I_{j}} w_{i j}
$$

If there are no multi-gene associated CpGs, then $N_{j}$ is simply the number of probes measured across gene $j$.

3. Let $A$ define the set of significant differentially methylated Cpgs. For each gene $j$, define an indicator vector $\mathbf{1}_{j}(x)$ of length $I_{j}$ such that $x_{i}=1$ if $C p G_{i j} \in A$, and $x_{i}=0$ if $C p G_{i j} \quad A$, where $i=1, \ldots, I_{j}$.

4. Let $\boldsymbol{w}_{j}$ define the vector of weights $w_{i j}$ for each gene $j$. Calculate the differential methylation score for each gene $j$

$$
S_{j}=\min \left(\mathbf{1}_{j}(x) \cdot \mathbf{w}_{j}, 1\right) .
$$

Note the maximum value of $S_{j}$ is 1 , and $S_{j}<1$ only in cases where the significant CpGs are all multi-gene associated, and the summed weights are less than one. $S_{j}=0$ when there are no significant differentially methylated CpGs across gene $j$.

5. Let $j=1, \ldots, J_{g}$ denote the genes that are present in gene set $g$. Calculate the enrichment statistic for Wallenius' noncentral hypergeometric test for each gene set $g$

$$
E S_{g}=\sum_{j=1}^{J_{g}} S_{j}
$$

For the standard Wallenius' noncentral hypergeometric test, the enrichment statistic is the intersection between the significant differentially methylated genes and genes in gene set $g$, which ignores multi-gene associated CpGs. Our modified enrichment statistic $E S_{g}$ accounts for multi-gene associated CpGs for gene set $g$.

6. Calculate the probability weighting function (PWF) by applying a moving average smoother to an ordered binary vector (based on the number of associated CpGs) where 1 indicates a gene is differentially methylated and 0 indicates the gene is not 
differentially methylated. We use the "tricubeMovingAverage" function in the limma package which is similar to a least squares loess curve of degree zero. The binary vector is ordered by the number of equivalent CpGs measuring methylation across each gene, $N_{j}$, from smallest to largest. The output is a vector of the same length as the input such that each gene is assigned a probability of differential methylation based on the smoothed value. We then calculate the expected odds of enrichment for each gene set $g$ by calculating the mean PWF of the genes in the set and comparing it to the mean PWF of the rest of the genes represented on the array.

$O D D S_{g}=\frac{\text { mean }(P W F(\text { genes in gene set } g))}{\text { mean }(P W F(\text { genes not in gene set } g))}$

7. For testing enrichment of each gene set $g$, we obtain a one-sided $\mathrm{p}$ value from Wallenius' noncentral hypergeometric distribution with the following parameters: $x=$ floor $\left(E S_{g}\right), m_{1}=$ the size of the gene set $J_{g}, m_{2}=$ the number of genes on the rest of the array, $n=$ the total number of significant genes, and odds $=O D D S_{g}$. We use the BiasedUrn R package to obtain $\mathrm{p}$ values.

\section{Null simulations: random sampling of $\mathrm{CpGs}$}

We randomly selected sets of 50,100, 500, 1000, 5000, and 10,000 CpGs from the Illumina array annotation for both 450k and EPIC arrays. The sampling was repeated 100 times for each CpG set size. We tested for significant enrichment of GO categories using a standard hypergeometric test (HGT), a Wallenius' hypergeometric test accounting for probe number bias (HGT-mod) and GOmeth, which is based on Wallenius' hypergeometric test and accounts for probe number and multi-gene bias.

\section{Methylation datasets}

The normal samples from the KIRC TCGA dataset [39] were used for estimating the false discovery rate of the different gene set testing methods. The data was downloaded using the curatedTCGAData Bioconductor package [40] and the 160 normal samples extracted. The data was provided as already processed $\beta$ values; however, we performed additional filtering and removed poor quality probes, probes containing SNPs as well as sex chromosome probes. The resulting multidimenational scaling plots showed no apparent evidence of sex or other technical effects (Fig. 3C).

In addition, 85 normal samples from the BRCA TCGA dataset [41] were used to estimate the false discovery rates of the seven gene set testing methods. The data was downloaded using the curatedTCGAData Bioconductor package and the 97 normal samples extracted. Following quality control 12 samples were removed (8 with unusual beta value distributions and 4 African/African American samples). Poor quality probes and probes containing SNPs were filtered out. Probes located on the sex chromosomes were retained as all the samples were female (Additional file 1: Fig. S3C).

To compare the performance between different gene set testing methods when there is significant differential methylation, we used Illumina Infinium HumanMethylationEPIC (GSE110554) data generated from flow-sorted neutrophils (Neu, $\mathrm{n}=6$ ), monocytes (Mono, $\mathrm{n}=6$ ), B-lymphocytes (B cells, $\mathrm{n}=6$ ), CD4+ T cells (CD4T, $\mathrm{n}=7$, six samples and one technical replicate), CD8+ T cells $(\mathrm{CD} 8 \mathrm{~T}, \mathrm{n}=6)$, natural killer cells $(\mathrm{NK}, \mathrm{n}=$ 
6) and 12 DNA artificial mixtures (labeled as MIX) [29]. Only the sorted cells were used in our analysis. The data was downloaded using the ExperimentHub Bioconductor package.

We also analyzed a developing B cell dataset that had matched DNA methylation and gene expression measurements [42]. Four populations of early B cell developmental stages were obtained from human fetal bone marrow, from 8 individuals. Methylation was measured using the Illumina HumanMethylation450 Beadchip, and gene expression was measured using the GeneChip Human Gene 1.0 ST Array (Affymetrix). The four populations were identified using flow cytometry antibodies and consist of Stage 1 (predominantly multipotent progenitors and common lymphoid progenitors), Stage 2 (pre-B-I cells), Stage 3 (pre-B-II cells) and Stage 4 (immature B cells). The data was downloaded from the Gene Expression Omnibus (GSE45461).

\section{Analysis and processing}

The majority of the analysis was performed using $\mathrm{R}$ (4.0.3) and some using $\mathrm{R}$ (3.6.1) [43]. The specific $R$ and package versions used for different aspects of the analysis can be viewed under the "Session information" sections of the analysis website associated with this study: http://oshlacklab.com/methyl-geneset-testing/.

\section{Quality control and normalization}

All methylation data was processed using the minfi [3, 44] R Bioconductor $[45,46]$ package. Between array and probe-type normalization was performed using the stratified quantile normalization (SQN) method [47]. Probes with a detection P value $>0.01$ in one or more samples were discarded. Probes potentially affected by common SNPs (minor allele frequency $>0$ ) proximal to the $\mathrm{CpG}$ of interest (up to $2 \mathrm{bp}$ upstream and 1 downstream) and non-specific probes [37, 48] were also removed from further analysis.

\section{Statistical analysis}

The proportion of methylation at each CpG is represented by the $\beta$ value, defined as the proportion of the methylated signal to the total signal and calculated from the normalized intensity values. Statistical analyses were performed on $M$ values $\left[M=\frac{\text { methylated }}{\text { unmethylated }}\right]$ as recommended by $\mathrm{Du}$ et al. [49].

\section{Comparison of gene set testing methods using sorted blood cell data}

CpG probe-wise linear models were fitted to determine differences in methylation between cell types (B cells vs NK, CD4 vs CD8 T cells, monocytes vs neutrophils) using the limma package [2]. Differentially methylated probes (DMPs) were identified using empirical Bayes moderated $t$ tests [50], performing robust empirical Bayes shrinkage of the gene-wise variances to protect against hypervariable probes [51]. Empirical Bayes moderated-t p values were then calculated relative to a minimum meaningful log-fold-change (lfc) threshold on the $\mathrm{M}$-value scale (lfc $=0.5$, corresponding to $|\Delta \beta| \sim 0.1$ ) [30]. P values were adjusted for multiple testing using the Benjamini-Hochberg procedure [52]. 
For each comparison, we tested for significant enrichment of GO categories and KEGG pathways. We only tested sets with at least 5 genes and at most 5000 genes for the methylGSA methods. The top ranked $5000 \mathrm{CpGs}$ were tested using the HGT and GOmeth, from the missMethyl package, for enrichment of GO terms and KEGG pathways. The raw $\mathrm{p}$ values were passed as input to the methylGSA methods. The ebGSEA methods were run using the ebGSEA R package (https://github.com/aet21/ebGSEA).

\section{Comparison of gene set testing methods using kidney clear cell carcinoma (KIRC) data}

The KIRC data [39] from the curatedTCGAData package was provided as $\beta$ values with masked data points; data points were masked as "NA" if their detection p value was greater than 0.05 or the probe was annotated as having a SNP within 10 base pairs or repeat within 15 base pairs of the interrogated CpG [53]. We extracted only the 160 normal samples and removed probes with any NA values, as well as SNP-affected probes and multi-mapping and sex-chromosome probes, as previously described. This left 364,602 probes for downstream analysis.

We ran 100 null simulations by randomly subsampling the normal samples and splitting them into two artificial "groups" with 5, 10, 20, 40, and 80 samples per group. For each of the 100 simulations, at each sample size, DMPs between groups were identified using empirical Bayes moderated t tests [50], performing robust empirical Bayes shrinkage of the gene-wise variances to protect against hypervariable probes [51].

We then performed gene set testing of the differential methylation analysis results using several methods with the Broad MSigDB gene sets available in the ChAMP Bioconductor package. GOmeth was run using both the top 1000 and top 5000 significant CpGs as input. The methylGSA methods; mGLM, mRRA (ORA), and mRRA (GSEA) were run with gene set sizes restricted to a minimum of 5 and maximum of 5000 genes. The ebGSEA method was run using default parameters and both its KPMT and WT output were compared.

\section{Comparison of gene set testing methods using breast invasive carcinoma (BRCA) data}

As with the KIRC data, the BRCA data [41] was downloaded using the curatedTCGAData package. We then extracted the 97 normal samples and removed probes with any NA values, as well as SNP-affected probes and multi-mapping probes, as previously described. Sex chromosome probes were retained since all sample donors were female. This left 371,389 probes for further analysis. Twelve outlying samples were removed (8 with unusual beta value distributions and 4 African/African American samples), leaving 85 samples for downstream analysis.

We ran 100 null simulations by randomly subsampling the normal samples and splitting them into two artificial groups with 5, 10, 20, and 40 samples per group. DMPs between the two groups were identified as described for the KIRC data, followed by the same gene set testing approach using the seven different methods previously outlined.

\section{RNA-Seq data and analysis}

The RNA-Seq data for the sorted blood cell types was downloaded from SRA (GSE107011; SRP125125) [31, 32]. The reads were mapped to hg19 reference transcriptome (http://refgenomes.databio.org/v2/asset/hg19_cdna/fasta/archive?tag=default) and 
quantified using Salmon (1.2.1) [54]. Salmon transcript-level estimates were imported and summarized at the gene-level as length-scaled TPM using the tximport Bioconductor package [55]. Lowly expressed genes were filtered out using the edgeR [56] "filterByExpr" function as described by Chen at al [57].. The data was then TMM normalized [58] and transformed using "voomWithQualityWeights" [59], to increase power by combining "voom" [60] observational-level weights with sample-specific weights.

Probe-wise linear models were then fitted for each gene to determine gene expression differences between cell types (B cells vs NK cells, CD4 vs CD8 T cells, monocytes vs neutrophils) using limma [2]. Differentially expressed genes were identified using empirical Bayes moderated $t$ tests [50], performing robust empirical Bayes shrinkage of the gene-wise variances to protect against hypervariable probes [51]. P values were adjusted for multiple testing using the Benjamini-Hochberg procedure [52].

We used the goana function from the limma package to test enrichment of GO categories, kegga to test for enrichment of KEGG pathways and a generalized version of goana and kegga; to test for enrichment of the Broad MSigDB gene sets. All the methods took gene length bias into account [23]. GO, KEGG and MSigDB truth sets were then defined for each cell type comparison from the RNA-Seq analysis as the top 100 enriched sets.

\section{Affymetrix array gene expression data and analysis}

The Affymetrix Human Gene 1.0 ST Array gene expression data for pre-B cell development was downloaded from GEO (GSE45460) [42].

The raw CEL files were loaded and processed using the oligo Bioconductor package. The data was background corrected, normalized, and summarized using Robust Multichip Average (RMA) pre-processing [33, 34]. Only probes with a median intensity greater than 4.5 , in at least 7 samples, were retained. Transcript-cluster identifiers that mapped to multiple Entrez identifiers were filtered out, along with any probes that did not map to Entrez identifiers, leaving 19,494 genes for downstream analysis.

Probe-wise linear models were then fitted for each gene to determine gene expression differences between Stage 1 and Stage 2 of pre B cell development (Stage 1 vs Stage 2) using limma [2]. Differentially expressed genes were identified using empirical Bayes moderated $t$ tests [50], performing robust empirical Bayes shrinkage of the gene-wise variances to protect against hypervariable probes [51]. P values were adjusted for multiple testing using the Benjamini-Hochberg procedure [52]. Genes with a $\log _{2}$ fold change greater than 0.5 using TREAT [30] and FDR less than 0.05 were deemed to be differentially expressed.

The goana function from the limma package was used to test enrichment of GO categories and kegga to test for enrichment of KEGG pathways. GO and KEGG truth sets were then defined for the Stage 1 versus Stage 2 comparison from the gene expression analysis as the top 100 enriched sets.

\section{Evaluation of GOregion using flow sorted blood cell data}

The lllumina Infinium HumanMethylationEPIC (GSE110554) data generated from flow-sorted blood cells was used for identification of DMRs. The data was processed as previously described. DMRs between cell types (B cells vs NK cells, CD4 vs CD8 T 
cells, monocytes vs neutrophils) were identified using the DMRcate Bioconductor package [16]. The analysis was performed on $M$ values using default parameters. Downstream gene set testing was performed on a filtered list of DMRs with a mean $|\Delta \beta| \geq 0.1$ and at least 3 underlying CpGs.

GO terms were tested for enrichment of DMR-associated genes using goregion and a standard HGT, as implemented in the goana function from the limma Bioconductor package. A gene, as defined in the TxDb.Hsapiens.UCSC.hg19.knownGene Bioconductor package, was included in the list of genes to be tested using goana if it overlapped a DMR by at least $1 \mathrm{bp}$.

\section{Evaluation of GOregion using pre-B cell development data}

The lllumina Infinium HumanMethylation450 (GSE45459) data generated from 4 stages of pre B cell development was used for identification of DMRs. The data was processed as previously described. DMRs between Stage 1 and Stage 2 were identified using the DMRcate Bioconductor package [16]. The analysis was performed on $M$ values using default parameters. DMRs were not filtered prior to gene set testing.

GO terms were tested for enrichment of DMR-associated genes using goregion and a standard HGT, as previously described for the sorted blood cell data.

\section{Supplementary Information}

The online version contains supplementary material available at https://doi.org/10.1186/s13059-021-02388-x.

Additional file 1. Supplementary figures.

Additional file 2. Supplementary tables.

Additional file 3. Review history.

\section{Acknowledgements}

We would like to acknowledge Peter Langfelder for providing detailed code and simulations which helped us to discover and correct for multi-gene bias in our testing framework. We would also like to acknowledge all missMethyl users who have provided valuable bug reports and feedback.

Review history

The review history is available as Additional file 3.

\section{Peer review information}

Barbara Cheifet was the primary editor of this article and managed its editorial process and peer review in collaboration with the rest of the editorial team.

\section{Authors' contributions}

The original idea for gene set testing for methylation array data was conceived by AO. BP conceived the statistical model and wrote the core of the gometh and gsameth codes. JM conceived the idea of extending GOmeth to DMRs. BP and JM determined how to apply GOmeth to DMRs. JM wrote the GOregion code. JM performed all analysis and produced the figures and analysis website. BP and JM wrote the initial draft of the manuscript, which was revised by all authors. The authors read and approved the final manuscript.

\section{Authors' information}

Twitter handles: @JovMaksimovic (Jovana Maksimovic); @AliciaOshlack (Alicia Oshlack); @BelindaPhipson (Belinda Phipson).

\section{Funding}

BP is supported by an Emerging Leader Investigator Grant (GNT1175653) from the National Health and Medical Research Council (NHMRC). AO is supported by NHMRC GNT1126157. The funding body did not play any role in the study design, analysis, interpretation of data or writing of the manuscript.

\section{Availability of data and materials}

All data used in this manuscript is publicly available. The KIRC [39] and BRCA [41] data was downloaded using the curatedTCGAData R Bioconductor package. The sorted blood cell type Illumina Infinium HumanMethylationEPIC data is available from the Gene Expression Omnibus under accession number GSE110554 [29] and was downloaded using the ExperimentHub R Bioconductor package. The RNA-Seq data for the sorted blood cell types was downloaded from SRA 
(GSE107011; SRP125125) [31, 32]. The methylation and gene expression datasets for the sorted B cell development data was downloaded from the Gene Expression Omnibus under accession number GSE45461 [42]. All analyses presented in this manuscript can be viewed at the following workflowr website: http://oshlacklab.com/methyl-geneset-testing/. All of the code used in these analyses is covered by the MIT License and is available from GitHub: https://github.com/ Oshlack/methyl-geneset-testing (DOI: 10.5281/zenodo.4777548) [61, 62].

\section{Declarations}

\section{Ethics approval and consent to participate}

Not applicable.

\section{Consent for publication}

Not applicable.

\section{Competing interests}

The authors declare that they have no competing interests.

\section{Author details}

${ }^{1}$ Peter MacCallum Cancer Centre, Melbourne, Victoria 3000, Australia. ${ }^{2}$ Department of Pediatrics, University of Melbourne, Parkville, Victoria 3010, Australia. ${ }^{3}$ Murdoch Children's Research Institute, Parkville, Victoria 3052, Australia. ${ }^{4}$ School of Biosciences, University of Melbourne, Parkville, Victoria 3010, Australia. ${ }^{5}$ Sir Peter MacCallum Department of Oncology, University of Melbourne, Parkville, Victoria 3010, Australia.

\section{Received: 25 August 2020 Accepted: 24 May 2021}

Published online: 08 June 2021

\section{References}

1. Esteller M. Cancer epigenomics: DNA methylomes and histone-modification maps. Nat Rev Genet. 2007;8(4):286-98. https://doi.org/10.1038/nrg2005.

2. Ritchie ME, Phipson B, Wu D, Hu Y, Law CW, Shi W, et al. limma powers differential expression analyses for RNAsequencing and microarray studies. Nucleic Acids Res. 2015;43(7):e47.

3. Aryee MJ, Jaffe AE, Corrada-Bravo H, Ladd-Acosta C, Feinberg AP, Hansen KD, et al. Minfi: a flexible and comprehensive Bioconductor package for the analysis of Infinium DNA methylation microarrays. Bioinformatics. 2014;30(10):1363-9. https://doi.org/10.1093/bioinformatics/btu049.

4. Phipson B, Maksimovic J, Oshlack A. missMethyl: an R package for analyzing data from Illumina's HumanMethylation450 platform. Bioinformatics. 2016;32(2):286-8. https://doi.org/10.1093/bioinformatics/btv560.

5. Davis S, Du P, Bilke S, Triche T Jr, Bootwalla M. methylumi: handle Illumina methylation data; 2019.

6. Pidsley R, Wong CCY, Volta M, Lunnon K, Mill J, Schalkwyk LC. A data-driven approach to preprocessing Illumina 450K methylation array data. BMC Genomics. 2013;14(1):293.

7. Morris TJ, Butcher LM, Feber A, Teschendorff AE, Chakravarthy AR, Wojdacz TK, et al. ChAMP: 450k chip analysis methylation pipeline. Bioinformatics. 2014;30(3):428-30. https://doi.org/10.1093/bioinformatics/btt684.

8. Assenov Y, Müller F, Lutsik P, Walter J, Lengauer T, Bock C. Comprehensive analysis of DNA methylation data with RnBeads. Nat Methods. 2014;11(11):1138-40. https://doi.org/10.1038/nmeth.3115.

9. Müller F, Scherer M, Assenov Y, Lutsik P, Walter J, Lengauer T, et al. RnBeads 2.0: comprehensive analysis of DNA methylation data. Genome Biol. 2019;20(1):55.

10. Oytam Y, Sobhanmanesh F, Duesing K, Bowden JC, Osmond-McLeod M, Ross J. Risk-conscious correction of batch effects: maximising information extraction from high-throughput genomic datasets. BMC Bioinformatics. 2016;17(1):332. https://doi.org/10.1186/s12859-016-1212-5.

11. Xu Z, Niu L, Li L, Taylor JA. ENmix: a novel background correction method for Illumina HumanMethylation 450 BeadChip. Nucleic Acids Res. 2016;44(3):e20. https://doi.org/10.1093/nar/gkv907.

12. Eckhardt F, Lewin J, Cortese R, Rakyan VK, Attwood J, Burger M, et al. DNA methylation profiling of human chromosomes 6, 20 and 22. Nat Genet. 2006;38(12):1378-85. https://doi.org/10.1038/ng1909.

13. Hansen KD, Timp W, Bravo HC, Sabunciyan S, Langmead B, McDonald OG, et al. Increased methylation variation in epigenetic domains across cancer types. Nat Genet. 2011;43(8):768-75. https://doi.org/10.1038/ng.865.

14. Butcher LM, Beck S. Probe Lasso: a novel method to rope in differentially methylated regions with 450K DNA methylation data. Methods. 2015;72:21-8. https://doi.org/10.1016/j.ymeth.2014.10.036.

15. Jaffe AE, Murakami P, Lee H, Leek JT, Fallin MD, Feinberg AP, et al. Bump hunting to identify differentially methylated regions in epigenetic epidemiology studies. Int J Epidemiol. 2012;41(1):200-9. https://doi.org/10.1093/ije/dyr238.

16. Peters TJ, Buckley MJ, Statham AL, Pidsley R, Samaras K, Lord RV, et al. De novo identification of differentially methylated regions in the human genome. Epigenetics Chromatin. 2015;8(1):6.

17. Martorell-Marugán J, González-Rumayor V, Carmona-Sáez P. mCSEA: detecting subtle differentially methylated regions. Bioinformatics. 2019;35(18):3257-62. https://doi.org/10.1093/bioinformatics/btz096.

18. Rijlaarsdam MA, van der Zwan YG, Dorssers LCJ, Looijenga LHJ. DMRforPairs: identifying differentially methylated regions between unique samples using array based methylation profiles. BMC Bioinformatics. 2014;15(1):141. https://doi.org/1 0.1186/1471-2105-15-141.

19. Subramanian A, Tamayo P, Mootha VK, Mukherjee S, Ebert BL, Gillette MA, et al. Gene set enrichment analysis: a knowledge-based approach for interpreting genome-wide expression profiles. Proc Natl Acad Sci U S A. 2005;102(43): 15545-50. https://doi.org/10.1073/pnas.0506580102.

20. Yaari G, Bolen CR, Thakar J, Kleinstein SH. Quantitative set analysis for gene expression: a method to quantify gene set differential expression including gene-gene correlations. Nucleic Acids Res. 2013;41(18):e170. https://doi. org/10.1093/nar/gkt660. 
21. Wu D, Lim E, Vaillant F, Asselin-Labat M-L, Visvader JE, Smyth GK. ROAST: rotation gene set tests for complex microarray experiments. Bioinformatics. 2010;26(17):2176-82. https://doi.org/10.1093/bioinformatics/btq401.

22. Wu D, Smyth GK. Camera: a competitive gene set test accounting for inter-gene correlation. Nucleic Acids Res. 2012; 40(17):e133. https://doi.org/10.1093/nar/gks461.

23. Young MD, Wakefield MJ, Smyth GK, Oshlack A. Gene ontology analysis for RNA-seq: accounting for selection bias. Genome Biol. 2010;11(2):R14. https://doi.org/10.1186/gb-2010-11-2-r14.

24. Dong D, Tian Y, Zheng SC, Teschendorff AE. ebGSEA: an improved gene set enrichment analysis method for epigenome-wide-association studies. Bioinformatics. 2019;35(18):3514-6. https://doi.org/10.1093/bioinformatics/ btz073.

25. Ren X, Kuan PF. methylGSA: a Bioconductor package and Shiny app for DNA methylation data length bias adjustment in gene set testing. Bioinformatics. 2019;35(11):1958-9. https://doi.org/10.1093/bioinformatics/bty892.

26. Mi G, Di Y, Emerson S, Cumbie JS, Chang JH. Length bias correction in gene ontology enrichment analysis using logistic regression. PLoS One. 2012;7(10):e46128. https://doi.org/10.1371/journal.pone.0046128.

27. Zhang Y, Maksimovic J, Naselli G, Qian J, Chopin M, Blewitt ME, et al. Genome-wide DNA methylation analysis identifies hypomethylated genes regulated by FOXP3 in human regulatory T cells. Blood. 2013;122(16):2823-36. https://doi.org/1 0.1182/blood-2013-02-481788.

28. Phipson B, Oshlack A. DiffVar: a new method for detecting differential variability with application to methylation in cancer and aging. Genome Biol. 2014;15(9):465. https://doi.org/10.1186/s13059-014-0465-4.

29. Salas LA, Koestler DC, Butler RA, Hansen HM, Wiencke JK, Kelsey KT, et al. An optimized library for reference-based deconvolution of whole-blood biospecimens assayed using the Illumina HumanMethylationEPIC BeadArray. Genome Biol. 2018;19(1):64. https://doi.org/10.1186/s13059-018-1448-7.

30. McCarthy DJ, Smyth GK. Testing significance relative to a fold-change threshold is a TREAT. Bioinformatics. 2009;25(6): 765-71. https://doi.org/10.1093/bioinformatics/btp053.

31. Monaco G, Lee B, Xu W, Mustafah S, Hwang YY, Carré C, et al. RNA-Seq signatures normalized by mRNA abundance allow absolute deconvolution of human immune cell types. Cell Rep. 2019;26(6):1627-40 e7.

32. Xu W, Monaco G, Wong EH, Tan WLW, Kared H, Simoni Y, et al. Mapping of $\gamma / \delta$ T cells reveals V $\delta 2+T$ cells resistance to senescence. EBioMedicine. 2019;39:44-58. https://doi.org/10.1016/j.ebiom.2018.11.053.

33. Bolstad BM, Irizarry RA, Astrand M, Speed TP. A comparison of normalization methods for high density oligonucleotide array data based on variance and bias. Bioinformatics. 2003;19(2):185-93. https://doi.org/10.1093/bioinformatics/19.2.185.

34. Irizarry RA, Hobbs B, Collin F, Beazer-Barclay YD, Antonellis KJ, Scherf U, et al. Exploration, normalization, and summaries of high density oligonucleotide array probe level data. Biostatistics. 2003;4(2):249-64. https://doi.org/10.1093/biosta tistics/4.2.249.

35. Gardiner-Garden M, Frommer M. CpG islands in vertebrate genomes. J Mol Biol. 1987;196(2):261-82. https://doi.org/10.1 016/0022-2836(87)90689-9.

36. Vazquez Ml, Catalan-Dibene J, Zlotnik A. B cells responses and cytokine production are regulated by their immune microenvironment. Cytokine. 2015;74(2):318-26. https://doi.org/10.1016/j.cyto.2015.02.007.

37. Pidsley R, Zotenko E, Peters TJ, Lawrence MG, Risbridger GP, Molloy P, et al. Critical evaluation of the Illumina MethylationEPIC BeadChip microarray for whole-genome DNA methylation profiling. Genome Biol. 2016;17(1):208. https://doi.org/10.1186/s13059-016-1066-1.

38. Blischak JD, Carbonetto P, Stephens M. Creating and sharing reproducible research code the workflowr way. F1000Res. 2019:8:1749

39. Cancer Genome Atlas Research Network. Comprehensive molecular characterization of clear cell renal cell carcinoma. Nature. 2013;499(7456):43-9. https://doi.org/10.1038/nature12222.

40. Ramos M. curatedTCGAData: curated data from The Cancer Genome Atlas (TCGA) as MultiAssayExperiment Objects; 2020.

41. Cancer Genome Atlas Network. Comprehensive molecular portraits of human breast tumours. Nature. 2012;490(7418): 61-70. https://doi.org/10.1038/nature11412.

42. Lee S-T, Xiao Y, Muench MO, Xiao J, Fomin ME, Wiencke JK, et al. A global DNA methylation and gene expression analysis of early human B-cell development reveals a demethylation signature and transcription factor network. Nucleic Acids Res. 2012;40(22):11339-51. https://doi.org/10.1093/nar/gks957.

43. R Core Team. R: A language and environment for statistical computing [Internet]. Vienna, Austria: R Foundation for Statistical Computing; 2014. Available from: http://www.r-project.org/

44. Fortin J-P, Triche TJ Jr, Hansen KD. Preprocessing, normalization and integration of the Illumina HumanMethylationEPIC array with minfi. Bioinformatics. 2017;33(4):558-60. https://doi.org/10.1093/bioinformatics/btw691.

45. Gentleman RC, Carey VJ, Bates DM, Bolstad B, Dettling M, Dudoit S, et al. Bioconductor: open software development for computational biology and bioinformatics. Genome Biol. 2004;5(10):R80. https://doi.org/10.1186/gb-2004-5-10-r80.

46. Huber W, Carey VJ, Gentleman R, Anders S, Carlson M, Carvalho BS, et al. Orchestrating high-throughput genomic analysis with Bioconductor. Nat Methods. 2015;12(2):115-21. https://doi.org/10.1038/nmeth.3252.

47. Touleimat N, Tost J. Complete pipeline for Infinium( $\left(^{(}\right)$Human Methylation 450K BeadChip data processing using subset quantile normalization for accurate DNA methylation estimation. Epigenomics. 2012;4(3):325-41. https://doi.org/10.2217/ epi.12.21.

48. Chen Y-A, Lemire M, Choufani S, Butcher DT, Grafodatskaya D, Zanke BW, et al. Discovery of cross-reactive probes and polymorphic CpGs in the Illumina Infinium HumanMethylation450 microarray. Epigenetics. 2013;8(2):203-9. https://doi. org/10.4161/epi.23470.

49. Du P, Zhang X, Huang C-C, Jafari N, Kibbe WA, Hou L, et al. Comparison of Beta-value and M-value methods for quantifying methylation levels by microarray analysis. BMC Bioinformatics. 2010;11(1):587.

50. Smyth GK. limma: Linear Models for Microarray Data. In: Gentleman R, Carey VJ, Huber W, Irizarry RA, Dudoit S, editors. Bioinformatics and computational biology solutions using R and bioconductor. New York, NY: Springer New York; 2005. p. $397-420$.

51. Phipson B, Lee S, Majewski IJ, Alexander WS, Smyth GK. Robust hyperparameter estimation protects against hypervariable genes and improves power to detect differential expression. Ann Appl Stat. 2016;10(2):946-63. https://doi. org/10.1214/16-AOAS920. 
52. Benjamini Y, Hochberg Y. Controlling the false discovery rate: a practical and powerful approach to multiple testing. J R Stat Soc Series B Stat Methodol. 1995;57(1):289-500.

53. Cancer Genome Atlas Research Network, Linehan WM, Spellman PT, Ricketts CJ, Creighton CJ, Fei SS, et al. Comprehensive molecular characterization of papillary renal-cell carcinoma. N Engl J Med. 2016;374(2):135-45.

54. Patro R, Duggal G, Love MI, Irizarry RA, Kingsford C. Salmon provides fast and bias-aware quantification of transcript expression. Nat Methods. 2017;14(4):417-9. https://doi.org/10.1038/nmeth.4197.

55. Soneson C, Love MI, Robinson MD. Differential analyses for RNA-seq: transcript-level estimates improve gene-level inferences. F1000Res. 2015;4:1521.

56. Robinson MD, McCarthy DJ, Smyth GK. edgeR: a Bioconductor package for differential expression analysis of digital gene expression data. Bioinformatics. 2010;26(1):139-40. https://doi.org/10.1093/bioinformatics/btp616.

57. Chen Y, ATL L, Smyth GK. From reads to genes to pathways: differential expression analysis of RNA-Seq experiments using Rsubread and the edgeR quasi-likelihood pipeline. F1000Res. 2016;5:1438.

58. Robinson MD, Oshlack A. A scaling normalization method for differential expression analysis of RNA-seq data. Genome Biol. 2010;11(3):R25. https://doi.org/10.1186/gb-2010-11-3-r25.

59. Liu R, Holik AZ, Su S, Jansz N, Chen K, Leong HS, et al. Why weight? Modelling sample and observational level variability improves power in RNA-seq analyses. Nucleic Acids Res. 2015;43(15):e97. https://doi.org/10.1093/nar/gkv412.

60. Law CW, Chen Y, Shi W, Smyth GK. voom: precision weights unlock linear model analysis tools for RNA-seq read counts. Genome Biol. 2014;15(2):R29.

61. Maksimovic J, Oshlack A, Phipson B. Gene set enrichment analysis for genome-wide DNA methylation data [Internet]. GitHub. Available from: https://github.com/Oshlack/methyl-geneset-testing.

62. Maksimovic J, Oshlack A, Phipson B. Gene set enrichment analysis for genome-wide DNA methylation data [Internet]. Zenodo. Available from: https://doi.org/10.5281/zenodo.4777548

\section{Publisher's Note}

Springer Nature remains neutral with regard to jurisdictional claims in published maps and institutional affiliations.

\section{Ready to submit your research? Choose BMC and benefit from:}

- fast, convenient online submission

- thorough peer review by experienced researchers in your field

- rapid publication on acceptance

- support for research data, including large and complex data types

- gold Open Access which fosters wider collaboration and increased citations

- maximum visibility for your research: over 100M website views per year

At $\mathrm{BMC}$, research is always in progress. 


\section{University Library}

\section{- M M I N E R VA A gateway to Melbourne's research publications}

Minerva Access is the Institutional Repository of The University of Melbourne

Author/s:

Maksimovic, J;Oshlack, A;Phipson, B

Title:

Gene set enrichment analysis for genome-wide DNA methylation data

Date:

2021-06-08

Citation:

Maksimovic, J., Oshlack, A. \& Phipson, B. (2021). Gene set enrichment analysis for genome-wide DNA methylation data. GENOME BIOLOGY, 22 (1), https://doi.org/10.1186/ s13059-021-02388-X.

Persistent Link:

http://hdl.handle.net/11343/278448

License:

CC BY 